\title{
Detection of inhibition of antimicrobial activity by mycobacterial lysates in human monocytes infected with Legionella pneumophila
}

\author{
Octavio M. Rivero-Lezcano ${ }^{\mathrm{a}}$, Leandro B. Rodríguez-Aparicio ${ }^{\mathrm{b}}$
}

${ }^{a}$ Unit of Investigation, Hospital of León, León, Spain

${ }^{\mathrm{b}}$ Molecular Biology Department, University of León, Campus de Vegazana, León, Spain

Abbreviations: BCYE, buffered charcoal yeast lysate; ATTC, American Type Culture Collection; CFU, colony forming units; CECT, Spanish Type Culture Collection; DAPI, 4,6,-DiAmidino-2-PhenylIndole; IFN- $\gamma$, interferon gamma; IL, interleukin; OADC, oleic acid-bovine albumin-dextrose-catalase; PBS, phosphate buffered saline; SD, standard deviation; TNF- $\alpha$, tumor necrosis factor $\alpha$.

* Corresponding author. Unit of Investigation, Hospital de León. Bldg. S. Antonio Abad. Altos de Nava s/n, 24008-León (Spain) Tel.: +34 987234041

E-mail address: orivero@hleo.sacyl.es 


\begin{abstract}
No in vitro antimicrobial activity has been detected in human monocytes infected with $\underline{\text { Mycobacterium tuberculosis, }}$, and the molecular mechanisms allowing the bacteria to survive intracellularly are unknown. As a means to test the influence of bacterial products in the microbicidal activity of monocytes we have developed an infection model with Legionella pneumophila, which is killed by interferon gamma activated cells. We demonstrate that this model is useful because $\underline{M}$. tuberculosis lysates inhibit one hundred fold the interferon gamma induced activity against $\underline{\text { L. pneumophila. }}$ Comparable degrees of inhibition, however, are detected when we use lysates from the less pathogenic M. gordonae, the pathogenic Staphylococcus aureus and the nonpathogenic Escherichia coli, suggesting the participation of a common mechanism. This hypothesis is supported by the fact that the pattern of cytokine secretion is similar in all cases. A significant difference is, however, observed for E. coli lysates, which induce the cell death of $\underline{L}$. pneumophila infected monocytes.
\end{abstract}

Keywords: Mycobacterium tuberculosis, Legionella pneumophila, monocytes, apoptosis, cytokines 


\section{Introduction}

The pathogen responsible for pulmonary tuberculosis, Mycobacterium

$\underline{\text { tuberculosis, }}$, is initially ingested by the alveolar macrophages in the lung (Raja, 2004), but we do not fully understand its virulence mechanisms in this cellular host. It does not produce toxins, and several candidate molecules, among others extracellular proteins and cell surface components, have been proposed as necessary for the intracellular survival (Smith, 2003). Nevertheless, none of them is currently acknowledged to have a more important role than the others. The influence of these molecules in the antimycobacterial mechanisms of human monocytes is difficult to test in $\underline{\mathrm{M}}$.

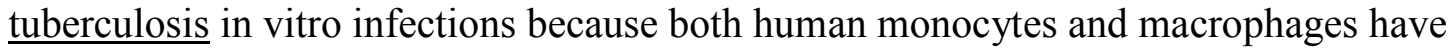
failed to show microbicidal activity against the bacteria (Fenton and Vermeulen, 1996; Raja, 2004). For this reason we have looked for other bacterial models suitable to perform these kind of tests.

Legionella pneumophila is also a pulmonary intracellular pathogen, which is responsible for Legionnaires'disease. While conditions are favourable L. pneumophila replicates intracellularly, but when they become difficult, the bacteria expresses traits that permit escape from the host cell and the transmission to a new host (Molmeret et al., 2004). Innate immunity to L. pneumophila infection also involve their phagocytosis by alveolar macrophages. Subsequent adaptive immunity is developed by TH1 cells (Friedman et al., 2002).

Murine macrophages are activated with IFN- $\gamma$ to kill $\underline{\mathrm{M}}$. tuberculosis, but this cytokine does not succeed to induce human macrophages to restrict the multiplication of the bacteria (Douvas et al., 1985). Although there are many evidences that support its essential role in human tuberculosis (Ottenhoff et al., 2005), it is not effective in vitro. 


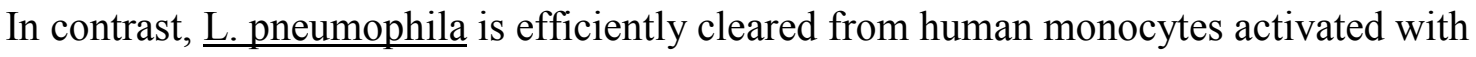
IFN- $\gamma$ (Bhardwaj et al., 1986).

We were interested in designing an assay which would allow us to check the influence of mycobacterial products on the process of IFN- $\gamma$ macrophage activation. We reasoned that the addition of the molecules of interest to infected macrophages would help us to select those that are able to inhibit the intracellular killing of bacteria. With

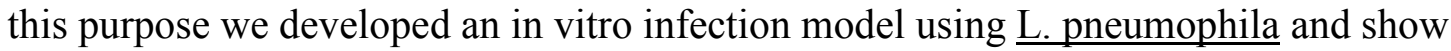
that it is appropriate to test bacterial lysates from both pathogenic and non-pathogenic mycobacteria.

\section{Materials and methods}

\subsection{Isolation of monocytes}

Peripheral blood was obtained from healthy volunteers following informed consent and approval of the protocol by the Hospital de León Clinical Research Ethics Board. Mononuclear cells were isolated by Ficoll-Paque Plus density gradient sedimentation (Amersham Biosciences). $\mathrm{CD} 14^{+}$cells (monocytes) were purified from mononuclear cells by magnetic cell separation (Myltenyi Biotec). The number of monocytes was calculated by counting in a Neubauer chamber and they were cultivated, within 4 hours from blood collection, in serum free media X-VIVO 15 without antibiotics (Cambrex Bio Science), at $37^{\circ} \mathrm{C}$ in $5 \% \mathrm{CO}_{2}$.

\subsection{Bacteria strains and bacterial lysates preparation}

L. pneumophila Philadelphia, ATCC 13151, was generously provided by Carmen Pelaz. A four days colony was suspended in PBS, spread in BCYE plates, and cultivated overnight. Next day, bacteria were lifted in X-VIVO 15 medium / 20\% glycerol, resuspended by repeated pippeting and frozen in single-use aliquots at $-80^{\circ} \mathrm{C}$. $\underline{\text { Staphylococcus aureus, ATCC25923 (purchased from ATCC) and Escherichia coli, }}$ 
CECT568 (purchased from CECT), were grown overnight in mannitol salt agar and Luria agar, respectively. Mycobacteria strains, which were cultivated before use for 10$15 \mathrm{~d}$ in Middlebrook 7H11 agar supplemented with Middlebrook enrichment OADC, were all isolated at the Hospital of León, and kindly provided by Julio Blanco and Manuela Caño (Microbiology Service). M. tuberculosis HL186T is a clinical isolate

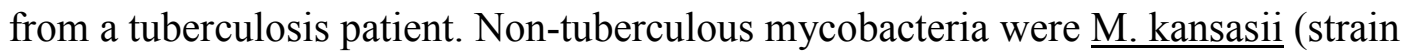
HL228K), M. avium (strain HL70A) and M. gordonae (strain HL184G).

For the preparation of bacterial lysates, colonies from fresh cultures in agar plates were lifted and suspended in X-VIVO $15(500 \mu \mathrm{l})$. They were sonicated using an S-450 digital ultrasonic cell disruptor (Branson). 12 pulses of $5 \mathrm{~s}$, with $10 \mathrm{~s}$ pauses, were applied in ice using a microtip at an amplitude of 70\% (25 Watts). $500 \mu \mathrm{l}$ of XVIVO 15 was added and sonicates were centrifuged at $4^{\circ} \mathrm{C}$ twice at $10,000 \times \mathrm{g}$ for 1 $\min$, once at $12,000 \times \mathrm{g}$ for $1 \mathrm{~min}$ and once at $15,000 \times \mathrm{g}$ for $3 \mathrm{~min}$. After each centrifugation supernatants were recovered, taking care to leave bacterial debris undisturbed. No live bacteria was recovered in the last supernatant, as demonstrated by inoculation of several aliquots in agar plates. Protein quantification of lysates was made with the Bio-Rad Protein Assay (Bio-Rad Laboratories). One use aliquots of 20-40 $\mu 1$ were frozen at $-30^{\circ} \mathrm{C}$.

\subsection{Monocyte infection}

Experiments were made in 96-well plates, always in a total volume of $100 \mu 1$. $10^{5}$ monocytes per well were infected with $\underline{L}$. pneumophila $\left(10^{2}\right.$ bacteria per well) in X-VIVO 15 supplemented with 10\% autologous unheated serum and, when indicated, $25 \mathrm{ng} / \mathrm{ml}$ of IFN- $\gamma\left(>2 \times 10^{7}\right.$ units/mg, Peprotech) were added. For cell treatments, 10 $\mu \mathrm{g}$ of protein from bacterial lysates were added to the infected monocytes. No washes were done at any moment. After three days, monocytes were lysed to release Legionella 
by sonication with a microtip (Branson), at an amplitude of $10 \%$ (2 Watts) for $3 \mathrm{~s}$. At this setting, ultrasounds were able to lyse monocytes without affecting Legionella viability. Decimal dilutions of the sonicates were inoculated in BCYE, incubated for 4 days, and the number of CFU was determined.

\subsection{Cell viability and apoptosis}

$5 \times 10^{4}$ Monocytes per well were allowed to adhere to 96 -well plates for 1 hour in RPMI medium without serum. RPMI was then substituted by X-VIVO 15 with 25 $\mathrm{ng} / \mathrm{ml}$ of IFN- $\gamma$. When indicated, the same medium with $10 \mu \mathrm{g}$ of the bacterial lysates were added and incubated for $3 \mathrm{~h}$ at $37^{\circ} \mathrm{C}$ in $5 \% \mathrm{CO}_{2}$. Cell viability was calculated by the trypan blue dye exclusion test in 500 cells, and results were expressed as the proportion of stained cells (non viable) from the total number of cells.

Proportion of apoptotic cells was calculated with the fluorescein FragEL DNA fragmentation kit (Calbiochem), which is based on the fluorescent labeling of DNA ends cleaved during apoptosis. $10^{5}$ cells were seeded on sterile $11 \mathrm{~mm}$ diameter cover glasses, and incubated in 24 well-plates in RPMI medium, as indicated in cell viability assays. After cells were treated with $40 \mu \mathrm{g}$ of bacterial lysates in $400 \mu \mathrm{l}$ of medium for 3

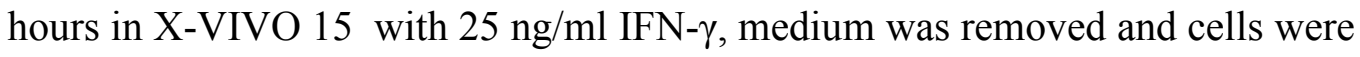
washed with PBS. Cells were fixed in 4\% formaldehyde/PBS for $15 \mathrm{~min}$ and conserved in $80 \%$ ethanol at $4^{\circ} \mathrm{C}$. Next day cells were labelled according to manufacturer's instructions, and the total number of cells was determined by DNA staining with DAPI. The proportion of apoptotic cells from 1,000 DAPI stained cells was determined.

\section{$\underline{2.5 \text { Quantitation of cytokines in supernatants }}$}

Culture supernatants from treated cells were recovered and frozen at $-80^{\circ} \mathrm{C}$. To remove remaining bacteria after thawing, samples were centrifuged at $4^{\circ} \mathrm{C}$ for $3 \mathrm{~min}$ at $3,000 \times \mathrm{g}$ in ultrafree-MC filter units of $0.45 \mu \mathrm{m}$ (Millipore). Cytokine determination 
was done by flow cytometry with the BD Cytometric Bead Array system (Becton Dickinson), using the Human Inflammation cytokine kit, which measures the amounts of IL-12, TNF- $\alpha$, IL-10, IL-6, IL-1 $\beta$ and IL-8. $50 \mu 1$ of each supernatant were processed following manufacturer's instructions, and cytokines were quantified as $\mathrm{pg} / \mathrm{ml}$ in a FACScan instrument (Becton Dickinson).

\subsection{Statistical analysis}

We determined that a log transformation of the variable CFU followed a normal distribution. To compare groups we performed a one-way analysis of variance and made pairwise comparisons by the Tukey's procedure. Cytokines distributions were not normal, and were analysed by the non-parametric Kruskal-Wallis method. We used Dunn's test for pairwise comparisons. In all cases, a p value $<0.05$ was considered significant. Analysis was performed with SPSS v. 14.0 and G-Stat v. 2.0 (Dunn's test).

\section{Results}

3.1. Mycobacterial lysates inhibit the antimicrobial activity of IFN- $\gamma$ activated monocytes infected with L. pneumophila

To confirm the ability of IFN- $\gamma$ to activate human monocytes we used the $\underline{L}$. pneumophila infection model. In stimulated monocytes, the evidence of bactericidal activity was the recovery of less bacteria $(\log C F U=0.64$ SD 0.87$)$ than the inoculated $(\log \mathrm{CFU}=1.88 \mathrm{SD} 0.27)$. In contrast, nonstimulated monocytes allowed a large level of intracellular multiplication $(\log \mathrm{CFU}=5.68 \mathrm{SD} 0.63$, Table I). Taking advantage of this model we aimed to answer two questions: first, whether mycobacterial lysates could antagonize the activating properties of IFN- $\gamma$ in human monocytes; and second, whether there were different degrees of monocyte inhibition depending on the bacterial species used to obtain the lysate.

The addition to the infected monocytes of lysates from $\underline{M}$. tuberculosis induced a partial inhibition of the IFN- $\gamma$ stimulation, and allowed the multiplication of $\underline{\mathrm{L}}$. 
pneumophila to $10^{4}$ bacteria per well, 100 fold more bacteria than the inoculated (Table I). This result demonstrated that the L. pneumophila infection model was appropriate to detect the inhibitory effect of lysates in the antimicrobial function of monocytes. To test the second question, whether the observed phenomenon was specific or not to $\underline{\mathrm{M}}$. $\underline{\text { tuberculosis, }}$, we prepared lysates from other mycobacteria, namely $\underline{\mathrm{M}}$. kansasii, $\underline{\mathrm{M}}$.

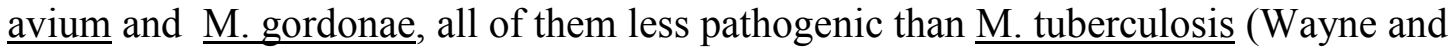
Sramek, 1992). Surprisingly, the same degree of inhibition was observed when their lysates were assayed ( $p>0.985$, Table I), and no relationship between the level of pathogenicity and the inhibition of monocytes could be established. An intriguing result

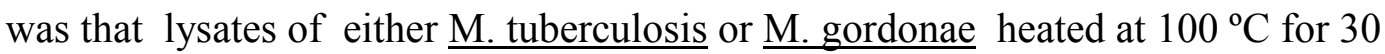
minutes were less inhibitory for stimulated monocytes but to a same degree, because we

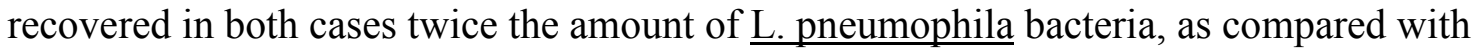
the untreated lysates. This result indicates that this effect is temperature sensitive and suggests that the nature of this inhibitory factor may be similar for both microorganisms (data not shown).

To extend the analysis of the specificity of the inhibitory effect to lysates from different species of bacteria, we included as additional controls lysates from the

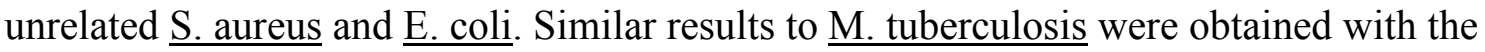

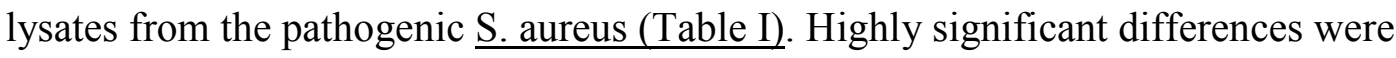
found, however, when lysates from $\underline{\mathrm{M} \text {. tuberculosis }}$ were compared to lysates from $\underline{\mathrm{E}}$.

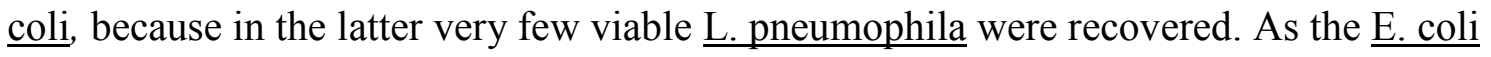
strain used was non-pathogenic, these results were not surprising. Under microscopic inspection, however, monocytes in the presence of E. coli lysates were not normal, and cells were apparently dead.

3.2. Lysates from E. coli, but not from mycobacteria or S. aureus, induce cell death 
Recovery of few viable bacteria from infections in the presence of both IFN- $\gamma$ and E. coli lysates may be a consequence of the bacterial products that did not antagonize the IFN- $\gamma$ stimulation. But another plausible reason is that E. coli lysates induced cell death, and L. pneumophila, an intracellular bacteria, will not multiply in a non viable host cell. This possibility prompted us to incubate non-infected monocytes activated with IFN- $\gamma$ in the presence of the various bacterial lysates and analyze both their viability and apoptotic status. As a negative control we included a culture without bacterial lysates.

We detected some apoptotic cells in the negative control (8.0\% SD 4.2), probably the consequence of manipulation during the cell purification and adherence in medium without serum . There were few differences, however, in the number of apoptotic cells when distinct lysates were used (Fig. 1). When we made pairwise comparisons between the number of apoptotic monocytes in the presence of the $\underline{\mathrm{M}}$. tuberculosis lysate with either the other bacteria lysates or the negative control, they did not reach statistical significance, with the exception of the lysate from E. coli. Although these results supported the previous microscopic observations, we did not believe that they fully explained the extensive cell death that was detected after an overnight incubation with E. coli lysates. Therefore, we also checked the viability of cells by trypan blue exclusion after three hours of incubation with bacterial lysates. Indeed, higher significance levels were found when we compared the proportion of non viable cells in E. coli lysates with either the negative control or with the use of any of the other lysates. From these results we concluded that E. coli lysates caused cell death in monocytes, mainly by a process of necrosis rather than apoptosis. We had previously confirmed that $\underline{L}$. pneumophila does no multiply extracellularly in X-VIVO 15 medium (data not shown), hence, they will not proliferate in cultures without live cells. 
3.3. Cytokine production profile is similar in supernatants from monocytes incubated with different bacterial lysates

We have not been able to find differences in the degree of inhibition induced by

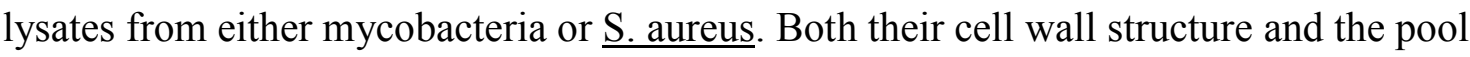
of proteins that they synthesize are, however, different. We wondered whether the reaction of monocytes to the presence of the lysates was variable, depending on the bacterial source. To find evidence about differences in the monocyte response to each of the lysates, we analysed in culture supernatants the secretion of the proinflammatory cytokines TNF- $\alpha$, IL-1 $\beta$, Il-6, IL-8 and IL-12, and the inhibitory IL-10. Monocytes did not produce IL-12 in any of the conditions tested, but when bacterial lysates were added, they secreted higher amounts of the rest of the cytokines than in the negative control, with the exception of IL-6 (Table II). Pairwise comparisons with the negative control were significant, however, only for TNF- $\alpha(\underline{\text { M. tuberculosis}})$ and IL-10 ( $\underline{\text { S. }}$ aureus). When comparisons were performed between the treatments with the different bacterial lysates no significant differences were found in the profiles induced. These results show that no specific pattern of cytokines in supernatants could be associated to the effect of a particular bacterial lysate.

\section{Discussion}

The member of the Mycobacterium genus of greater clinical importance is $\underline{\mathrm{M}}$. tuberculosis, but the features that make it so virulent as compared with other mycobacteria are unknown. Several molecules have been postulated as candidate virulence factors, and their importance has been tested by the construction of the corresponding knock out bacteria (Smith, 2003). This kind of assays, however, has not provided us with a conclusive explanation of the $\mathrm{M}$. tuberculosis virulence mechanisms (Barry, 2001). As a complementary strategy to determine the relative importance of 
these molecules, we devised an approach to measure modifications induced by mycobacterial products in the antimicrobial activity of human monocytes. It was important that the means to activate the cells was the addition of IFN- $\gamma$, because it is considered a critical cytokine in the resistance to tuberculosis (Ottenhoff et al., 2005). We also thought necessary not to use mycobacteria to quantify the antimicrobial activity because it has been described that IFN- $\gamma$ does not activate human monocytes to kill them (Douvas et al., 1985; Robertson and Andrew, 1991), so we have chosen a taxonomically unrelated bacterium, L. pneumophila.

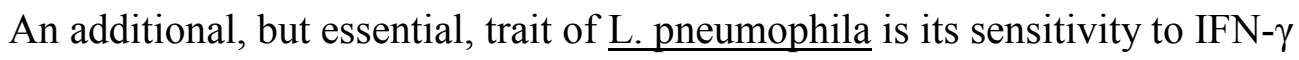
activated monocytes (Bhardwaj et al., 1986), which provides us with a sensitive assay. We made the infections with $10^{2}$ bacteria, a low multiplicity of infection of 0,001 bacterium per monocyte, which resembles more closely a natural infection, and which avoids the washing of non-phagocyted bacteria, diminishing the technical manipulations. After 3 days we obtained a large range of multiplication level, from $>$ $10^{5}$ bacteria in non stimulated monocytes to $<10^{1}$ bacteria when they were activated with IFN- $\gamma$. In our inhibition experiments we routinely recovered $10^{4}$ bacteria from the activated monocytes, but higher or lower degrees of inhibition would be technically easy to detect. In order to decrease the variability in the experiments we used a serumfree medium, with a consistent composition that do not depend on the addition of biologically variable components like foetal serum.

As a first step we initially used whole bacterial lysates, rather than purified molecules, which has the advantage of allowing the detection of activities that require the interactions between two or more different bacterial molecules. Furthermore, the exposure of infected macrophages to bacterial products has a biological meaning because, within the tuberculous granuloma, alveolar macrophages and freshly recruited 
monocytes are exposed to both live and killed bacteria (Houben et al., 2006). We observed that $\underline{\mathrm{M} \text {. tuberculosis }}$ lysates do inhibit the anti-Legionella activity of primary human monocytes stimulated with IFN- $\gamma$. From these results we concluded that the infection model was appropriate as a means to measure the influence of bacterial products on the IFN- $\gamma$ stimulated monocytes, although we found that this inhibitory effect was not specific for $\underline{\mathrm{M} \text {. tuberculosis }}$ (Table I). This assay, which is robust and shows little variability between experiments, may prove useful to learn about virulence mechanisms of pathogenic bacteria. Another strenght of this model is that it looks at one of the more important features of monocytes in infections, its antimicrobial activity. We may also use it to analyse the role of purified molecules from different origins, and to find both general and specific strategies for survival within the human host developed by different species of bacteria.

The use of crude extracts, however, do not allow us to know the reasons for the similarities in the effects induced by the lysates from bacteria with different degrees of pathogenicity. Nevertheless, it is possible that different bacterial molecules that bind the same kind of receptors in the monocyte surface, like toll-like receptors (Akira, 2003; Tsan and Gao, 2004), elicit comparable responses. This idea was supported by the cytokine profiles in supernatants, very similarly induced by all the bacterial lysates, and characteristic of an inflammatory response. Consequently, we postulate that the mechanisms of inhibition of monocytes depend on the release of cytokines which influence their own behavior. IL-6 produced by macrophages infected with $\underline{\mathrm{M}}$. tuberculosis, for example, has been shown to inhibit the antimicrobial activity of macrophages against the intracellular pathogen $\underline{\text { T. gondii }}$ (Nagabhushanam et al., 2003). In our conditions it is not likely that IL-6 is responsible for the inhibition, because it is also present in the negative control. Another candidate is the inhibitory IL- 
10 , but preliminary results of infections in the presence of a blocking anti-IL-10 antibody do not show differences in the number of bacteria recovered (data not shown).

Nevertheless, there were differences in the reaction of monocytes. The lysates of the non-pathogenic E. coli induced the cell death of monocytes. It has been described that different strains of E. coli induce cell death in macrophages by apoptotic and nonapoptotic mechanisms (Fernandez-Prada et al., 1998), and that purified LPS and Shiga toxin 1 from this bacteria also promote apoptosis (Harrison et al., 2005). The benefits of this effect for E. coli are not apparent. The removal of macrophages could be advantageous for an extracellular microorganism, but $\underline{\mathrm{S}}$. aureus, also extracellular, does not cause the same outcome.

In vitro, the lysates of the bacteria that we have tested induce the same basal level of inhibition in monocytes. In vivo, on the contrary, this is not sufficient for the non-pathogenic bacteria to cause disease in the human host. The clinical importance of this mechanism of inhibition might be, however, relevant for $\underline{\text { M. tuberculosis, }}$, because when it is killed in the tuberculous granuloma, large quantities of bacterial products are released.

In conclusion, the assay that we have developed has been proved useful to test the inhibition of the antimicrobial activity of activated monocytes by bacterial molecules, and may be used to characterize the molecular events that take place in the monocyte-pathogen interaction.

\section{Acknowledgments}

We thank the nurses that helped us with the blood collection. This study was supported by grants LE07/04 from Junta de Castilla y León, PI051288 from Instituto de Salud Carlos III-FIS (Plan Nacional de Investigación Científica, Desarrollo e Innovación Tecnológica) and BMC2003-03575 from Dirección General de 
Investigación. Dr. Rivero-Lezcano is a member of the Foundation Institute of Studies in Health Sciences and participates in the SACYL research program.

\section{References}

Akira, S., 2003. Mammalian Toll-like receptors. Curr. Opin. Immunol. 15, 5.

Barry, C. E. III, 2001. Interpreting cell wall 'virulence factors' of Mycobacterium tuberculosis. Trends Microbiol. 9, 237.

Bhardwaj, N., Nash, T. W., Horwitz, M. A.,1986. Interferon-gamma-activated human monocytes inhibit the intracellular multiplication of Legionella pneumophila. J. Immunol. 137, 2662.

Douvas, G. S., Looker, D. L., Vatter, A. E., Crowle, A. J., 1985. Gamma interferon activates human macrophages to become tumoricidal and leishmanicidal but enhances replication of macrophage-associated mycobacteria. Infect. Immun. 50, 1.

Fenton, M. J., Vermeulen, M. W., 1996. Immunopathology of tuberculosis: roles of macrophages and monocytes. Infect. Immun. 64, 683.

Fernandez-Prada, C., Tall, B. D., Elliott, S. E., Hoover, D. L., Nataro, J. P., Venkatesan, M. M., 1998. Hemolysin-positive enteroaggregative and cell-detaching Escherichia coli strains cause oncosis of human monocyte-derived macrophages and apoptosis of murine J774 cells. Infect. Immun. 66, 3918.

Friedman, H., Yamamoto, Y., Klein, T. W., 2002. Legionella pneumophila pathogenesis and immunity. Semin. Pediatr. Infect. Dis. 13, 273.

Harrison, L. M., Cherla, R. P., van den Hoogen, C., van Haaften, W. C., Lee, S. Y., Tesh, V. L., 2005. Comparative evaluation of apoptosis induced by Shiga toxin 1 and/or lipopolysaccharides in human monocytic and macrophage-like cells. Microb. Pathog. 38, 63. 
Houben, E. N., Nguyen, L., Pieters, J., 2006. Interaction of pathogenic mycobacteria with the host immune system. Curr. Opin. Microbiol. 9, 76.

Molmeret, M., Bitar, D. M., Han, L., Kwaik, Y. A., 2004. Cell biology of the intracellular infection by Legionella pneumophila. Microbes Infect. 6, 129.

Nagabhushanam, V., Solache, A., Ting, L. M., Escaron, C. J., Zhang, J. Y., Ernst, J. D., 2003. Innate inhibition of adaptive immunity: Mycobacterium tuberculosis-induced IL-6 inhibits macrophage responses to IFN-gamma. J. Immunol. 171, 4750.

Ottenhoff, T. H., Verreck, F. A., Hoeve, M. A., van de Vosse, E., 2005. Control of human host immunity to mycobacteria. Tuberculosis (Edinb) 85, 53.

Raja, A., 2004. Immunology of tuberculosis. Indian J. Med. Res. 120, 213.

Robertson, A. K., Andrew, P. W., 1991. Interferon gamma fails to activate human monocyte-derived macrophages to kill or inhibit the replication of a non-pathogenic mycobacterial species. Microb. Pathog. 11, 283.

Smith, I., 2003. Mycobacterium tuberculosis pathogenesis and molecular determinants of virulence. Clin. Microbiol. Rev. 16, 463.

Tsan, M. F., Gao, B., 2004. Endogenous ligands of Toll-like receptors. J. Leukoc. Biol. $76,514$.

Wayne, L. G., Sramek, H. A., 1992. Agents of newly recognized or infrequently encountered mycobacterial diseases. Clin. Microbiol. Rev. 5, 1. 
Table I. Effect of treatment with bacterial extracts on the multiplication of $\underline{L}$. pneumophila in human monocytes stimulated with interferon gamma.

\begin{tabular}{ll}
\hline Treatment & Log CFU $^{\mathrm{a}}$ \\
\hline Unstimulated monocytes & $5.68 \mathrm{SD} 0.63(5.02-6.35)^{*}$ \\
IFN- $\gamma$ stimulated monocytes & \\
No extract & 0.64 SD $0.87(-0.26-1.55)^{*}$ \\
$\underline{\text { M. tuberculosis lysate }}$ & 4.14 SD $0.68(3.42-4.85)$ \\
$\underline{\text { M. kansasii lysate }}$ & 3.96 SD $0.49(3.44-4.48)$ \\
$\underline{\text { M. avium lysate }}$ & 3.76 SD $1.08(2.41-5.10)$ \\
$\underline{\text { M. gordonae lysate }}$ & 4.15 SD $0.84(3.27-5.03)$ \\
$\underline{\text { E. coli lysate }}$ & 1.27 SD $0.41(0.83-1.71)^{*}$ \\
$\underline{\text { S. aureus lysate }}$ & 4.34 SD $0.16(4.09-4.59)$ \\
\hline
\end{tabular}

${ }^{a}$ Log number of CFU after 3 days in culture. Data represent mean and SD (95\% confident interval) for 6 experiments.

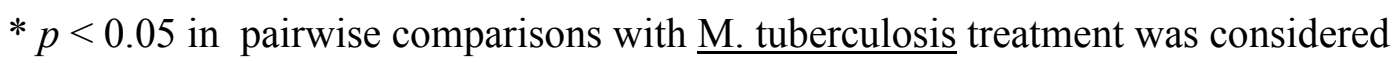
significant. 
Table II. Cytokine production by monocytes infected with $\underline{\text { L. pneumophila }}$ in the presence of lysates from different bacteria.

\begin{tabular}{lrrrrrr}
\hline Treatment & TNF- $\alpha$ & IL-1 $\beta$ & IL-6 & IL-8 & IL-10 & IL-12 \\
\hline No lysate & 412.5 & 141.0 & $2,891.3$ & $16,664.0$ & 26.8 & 0.0 \\
$\underline{\text { M. tuberculosis }}$ & $5,027.9^{*}$ & $15,753.0$ & $1,648.7$ & $104,914.4$ & $3,041.6$ & 0.0 \\
$\underline{\text { M. gordonae }}$ & $2,257.7$ & $15,357.4$ & $2,420.1$ & $104,202.1$ & $2,237.1$ & 0.0 \\
$\underline{\text { S. aureus }}$ & $4,714.7$ & $14,671.5$ & 848.3 & $176,321.8$ & $4,342.0^{*}$ & 0.0 \\
& & & & & &
\end{tabular}

Amount of cytokines produced after 3 days incubations in $\mathrm{pg} / \mathrm{ml}$. Data represent median for 3 experiments.

$* p<0.05$ in pairwise comparisons with "No lysate" treatment was considered significant 


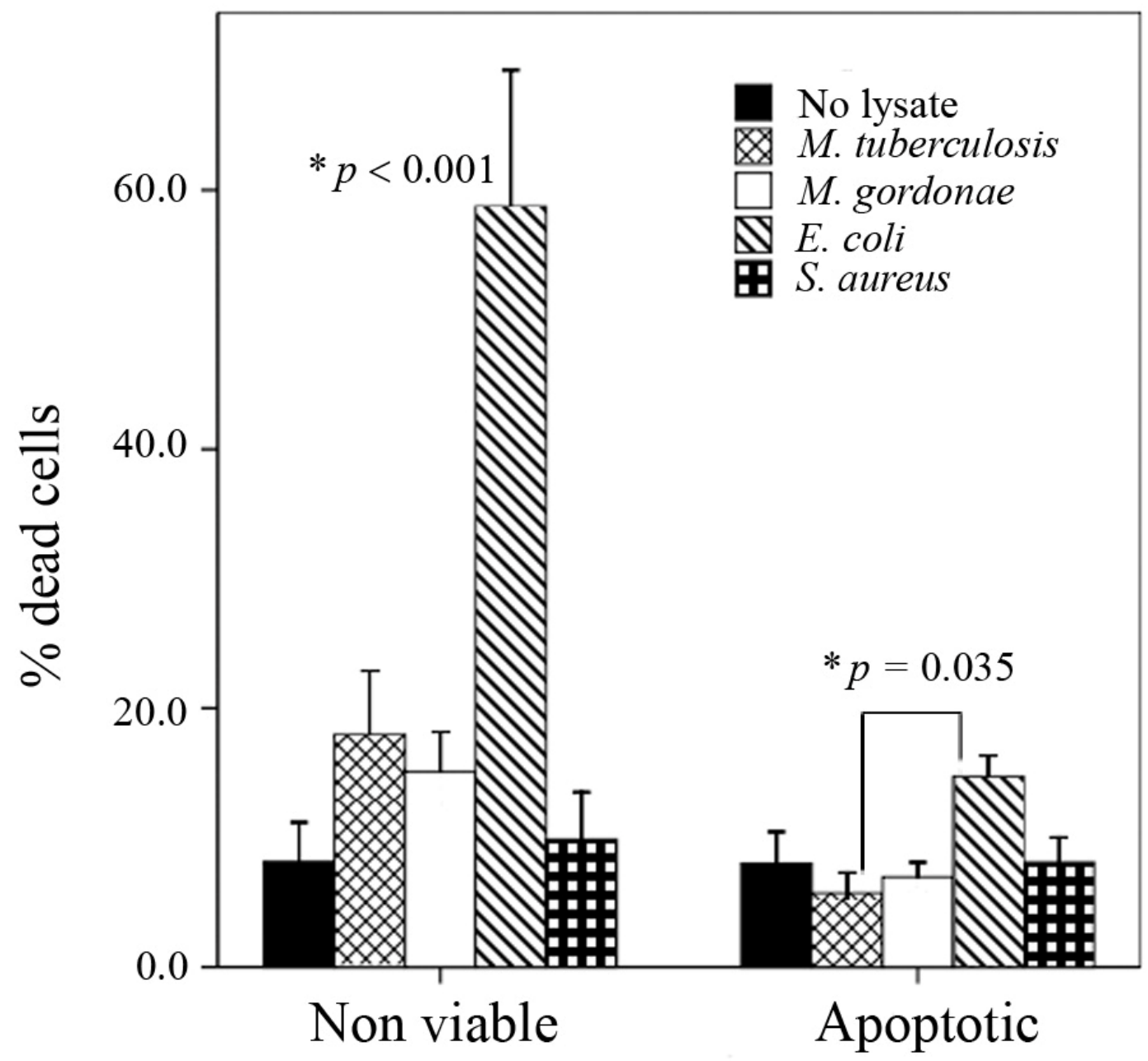

Fig. 1. Cell viability and apoptosis of monocytes incubated with bacterial lysates. Non viable cells were quantified by trypan blue exclusion and apoptotic cells by microscopic recognition of fluorescent labeled DNA cleaved in apoptosis. Data are the relative percent of dead cells to total number of cells and represent the mean $+\mathrm{SD}$ of four independent experiments. ${ }^{*} p<0.05$ was considered significant. 